\title{
Discrepancies between self- and observer- rated depression severities in patients with major depressive disorder associated with frequent emotion-oriented coping responses and hopelessness
}

This article was published in the following Dove Press journal: Neuropsychiatric Disease and Treatment

\author{
Emi Tsujimoto ${ }^{1,2}$ \\ Noa Tsujii' \\ Wakako Mikawa' \\ Hisae Ono² \\ Osamu Shirakawa' \\ 'Department of Neuropsychiatry, \\ Kindai University Faculty of \\ Medicine, Osakasayama, Osaka, Japan; \\ ${ }^{2}$ Department of Psychological Sciences, \\ Graduate School of Humanities, \\ Kwansei Gakuin University, \\ Nishinomiya, Hyogo, Japan
}

Purpose: The rating discrepancy for patients with major depressive disorder (MDD) is believed to be associated with hopelessness, risk of suicidal behavior, and personality characteristics, such as high neuroticism. However, it remains to be elucidated whether the discrepancy is also mediated by coping styles, which are conceptualized as personality characteristics.

Patients and methods: We enrolled 154 participants and divided them into three groups: patients with MDD with a rating discrepancy (MDD-WD; $n=46$ ), patients with MDD without a rating discrepancy (MDD-WoD; $\mathrm{n}=50$ ), and healthy controls ( $\mathrm{HCs} ; \mathrm{n}=58$ ). A rating discrepancy was defined as a high Beck Depression Inventory score and low Hamilton Depression Rating Scale score. Coping styles and hopelessness were compared among the groups.

Results: The MDD-WD group exhibited a higher level of hopelessness than those in the MDDWoD and HC groups. They also demonstrated a significantly increased number of suicide attempts compared with the MDD-WoD group. Both the MDD-WD and MDD-WoD groups exhibited lesser task-oriented and greater emotion-oriented coping styles than those in the HC group, with the MDD-WD group demonstrating even greater emotion-oribented coping than that in the MDDWoD group. Overall, high levels of hopelessness, a history of suicide attempts, and frequent use of emotion-oriented coping mechanisms were associated with rating discrepancy.

Conclusion: Patients with MDD who showed rating discrepancy tended to use emotion-oriented coping. Planning for minimal use of emotion-oriented coping may be a psychotherapeutic intervention for such patients. Reduced emotion-oriented coping may also reduce the feeling of hopelessness and risk of developing suicidal behavior.

Keywords: major depressive disorder, coping styles, emotion-oriented coping, self-rating, observer rating, hopelessness

\section{Introduction}

A self-recognized severity rating higher than the observer's rating for depression is believed to be one of the predictors of extreme hopelessness, suicidal behavior, and treatment resistance in major depressive disorder (MDD). ${ }^{1-3}$ This rating discrepancy in patients with MDD may be associated with personality styles or characteristics, such as high neuroticism, ${ }^{4,5}$ low extraversion, ${ }^{5,6}$ or low agreeableness. ${ }^{5}$ In addition, one recent investigation reported the association between rating discrepancy and affective temperament. ${ }^{7}$ However, it is still unknown whether such a discrepancy is
Correspondence: Noa Tsujii Kindai University Faculty of Medicine, 377-2 Ohnohigashi, Osakasayama,

Osaka 589-85II, Japan

$\mathrm{Tel}+8 \mid 723660221$

Fax +81723676950

Email tujiinoa@med.kindai.ac.jp 
also mediated by coping styles which are conceptualized as personality characteristics.

People in different situations are assumed to have preferences for different coping styles. Generally, adaptive coping refers to being flexible and efficient, whereas maladaptive coping refers to being rigid or socially inappropriate. The Coping Inventory for Stressful Situations (CISS), developed by Endler and Parker, ${ }^{8}$ is widely used in research on psychiatric disorders. It focuses on the identification and comparison of three basic coping strategies: 1) task-oriented coping; 2) emotion-oriented coping; and 3) avoidanceoriented coping. Task-oriented coping involves dealing directly with a stressful situation, emotion-oriented coping involves attending to the emotions surrounding the situation, and avoidance-oriented coping involves escape strategies in the form of distraction and social diversion.

Previous studies on coping styles in MDD have reported that task-oriented coping style is considered adaptive when a situation is manageable ${ }^{9}$ and is negatively associated with depression symptoms. ${ }^{10-13}$ In contrast, emotion- and avoidance-oriented coping styles are generally maladaptive for patients with MDD and are associated with depressive symptoms, ${ }^{12-16}$ anxiety, ${ }^{17}$ and current suicidality, ${ }^{18-21}$ suggesting that adaptive coping strategies reduce psychological distress whereas maladaptive coping styles are associated with depression and anxiety. ${ }^{22}$ Thus, the deleterious effects of MDD impair the capacity for effective coping. ${ }^{23}$

However, it is unknown whether coping styles of patients with MDD are associated with the discrepancy between selfand observer-rated depression severities; moreover, the ways in which these coping styles differ from those in healthy controls (HCs) remain unclear. Because both maladaptive coping styles and rating discrepancies are associated with suicidality, research on this association would be particularly beneficial for psychotherapeutic interventions in patients with MDD. Moreover, recent practice guidelines recommend both psychological and psychosocial interventions, particularly for the treatment of mild depression. Because planning an effective coping strategy is a type of psychotherapeutic intervention, understanding the association between rating discrepancies and coping styles could be critical for evaluating the efficacy of psychiatric interventions. We hypothesized that patients with MDD who rely on the use of maladaptive coping styles during stressful situations exhibit a discrepancy between self- and observer-rated depression severities, which may ultimately worsen the disease outcome. This study investigated how the aforementioned coping strategies are associated with discrepancies between self- and observerrated depression severities in patients with MDD.

\section{Patients and methods}

\section{Participants}

In total, 154 participants were included in this study. We recruited 96 patients who were a set of consecutive patients at the Kindai University Hospital and then divided them into two groups: patients with MDD with a rating discrepancy (MDD-WD group; $n=46$ ) and those with MDD without a rating discrepancy (MDD-WoD group; $\mathrm{n}=50$ ). The diagnosis of MDD was made according to the criteria outlined in the Diagnostic and Statistical Manual of Mental Disorders, fourth edition, using the Mental Illness Neuropsychiatric Interview (MINI Japanese version 5.0.0). ${ }^{24}$ All patients $(n=96)$ were categorized as having mild depression (scoring between 8 and 13 on the 17-item Hamilton Depression Rating Scale [HDRS]). ${ }^{25}$ Patients in the MDD-WD group had higher Beck Depression Inventory (BDI) scores ( $\geq 29$; considered to indicate severe depression), whereas those in the MDD-WoD group had lower BDI scores $(<29$; considered to indicate mild-tomoderate depression). HCs $(n=58)$ were also screened using MINI. Participants were excluded from the HC group if they had any history of psychiatric disorders or heritable neurological diseases among immediate or second-degree family members. In addition, a history of head trauma accompanied by the loss of consciousness, current or previous neurological disease, current or previous endocrine disease, bipolar disorder, schizophrenia, electroconvulsive therapy, and alcohol/substance abuse or addiction within the past 12 months were regarded as exclusion criteria. After presenting all the patients and participants with a complete description of the study, we obtained their written informed consent. This study complied with the Declaration of Helsinki and was approved by the ethics committee of Kindai University Faculty of Medicine (No 20-12).

\section{Clinical assessment}

Data regarding mental health service accessibility and use were not collected. The participants were assessed using BDI, HDRS, Beck Hopelessness Scale (BHS), and CISS on the same day. The observer's rating of depressive symptoms was obtained using the Japanese version of the Structured Interview Guide for the 17-item HDRS (SIGH-D). ${ }^{26}$ HDRS is the most widely used scale in the assessment of depression severity, and SIGH-D has been shown to improve the reliability of ratings. Self-rated depressive symptoms and signs were assessed using the Japanese version of BDI, second edition. ${ }^{27} \mathrm{BDI}$ includes 21 items and is also widely used in the assessment of depression severity. 
Level of hopelessness was assessed using BHS. ${ }^{28}$ BHS includes 20 items that assess negative attitudes about the future. Previous studies have demonstrated a significant association between BHS scores and suicidality. ${ }^{28}$

Coping strategies were evaluated according to the threefactor model using the Japanese version of CISS ${ }^{29}$ CISS consists of 48 items, each rated on a 5-point scale, which collectively assess three different coping styles: 1) task-oriented coping; 2) emotion-oriented coping; and 3) avoidanceoriented coping. Scores (range, 16-80) were generated for each coping style, with higher scores indicating a greater tendency to use the particular coping method.

\section{Statistical analysis}

Demographic and clinical variables were compared between the study groups using the chi-squared test for categorical variables and nonparametric Mann-Whitney $U$ test or Kruskal-Wallis one-way analysis of variance for continuous variables. If the Kruskal-Wallis test indicated significance, post hoc analysis was performed using pairwise comparisons of the groups with a Bonferroni-corrected alpha of 0.0167 (ie, 0.05/3). Furthermore, a stepwise logistic regression analysis was used to determine whether the coping styles and clinical variables independently predicted the discrepancies between self- and observer-rated depression severities in patients with MDD. For this analysis, the presence of such a discrepancy (converted to a binomial category; no $=0$, yes $=1$ ) was the dependent variable; potential confounding variables, such as age, $\operatorname{sex}($ male $=0$, female $=1)$, comorbid anxiety disorders (no $=0$, yes $=1)$, duration of illness, education level, history of suicide attempts (no $=0$, yes $=1$ ), BHS score, and each CISS domain, were controlled. Odds ratios with 95\% CIs were used to describe the observed associations. All statistical tests were performed using SPSS for Macintosh version 22.0 (IBM Corporation, Armonk, NY, USA). Statistical significance was assumed at $P<0.05$ (two-tailed).

\section{Results}

\section{Demographic and clinical characteristics}

The three study groups differed significantly with respect to HDRS, BDI, and BHS scores (all $P<0.001$; Table 1). Post hoc analysis indicated that the two MDD patient groups (MDD-WD and MDD-WoD) had significantly higher BHS scores than those of the HC group (both $P<0.001$ ). The MDD-WD group experienced a higher level of hopelessness and an increased number of suicide attempts than those by the MDD-WoD group $(P<0.001$ and $P=0.008$, respectively).

\section{Differences in stress-coping styles between the groups}

The three study groups differed significantly with respect to their preferred coping styles (task-oriented: $\chi^{2}=35.7$, $P<0.001$; emotion-oriented: $\chi^{2}=63.0, P<0.001$; avoidanceoriented: $\chi^{2}=8.65, P=0.013$; Figure 1). The two MDD patient groups had lower scores for task-oriented coping than those of the $\mathrm{HC}$ group (both $P<0.001$ ), but no significant

Table I Demographic and clinical characteristics

\begin{tabular}{|c|c|c|c|c|c|}
\hline & \multicolumn{3}{|c|}{ Patients with MDD } & \multirow[t]{2}{*}{ Statistics } & \\
\hline & WD $(n=46)$ & WoD $(n=50)$ & HCs $(n=58)$ & & \\
\hline & n (\%) & n (\%) & n (\%) & $\chi^{2}$ & \\
\hline Sex (female, \%) & $27(58.7)$ & $20(40.0)$ & $33(56.9)$ & 4.3 & \\
\hline Comorbid axis I disorder & $13(28.3)$ & $10(20.0)$ & & 0.9 & \\
\hline History of suicide attempts & $16(34.8)$ & $6(12.0)$ & & $7.0 *$ & \\
\hline Family history of psychotic disorders & $19(41.3)$ & $21(42.0)$ & & 0.005 & \\
\hline Family history of suicide & $8(17.4)$ & $6(12.0)$ & & 0.6 & \\
\hline Antidepressant use & $28(60.9)$ & $28(56.0)$ & & 0.2 & \\
\hline Mood stabilizer use & II (2.4) & II (22.0) & & 0.05 & \\
\hline \multirow[t]{2}{*}{ Antipsychotic drug use } & $14(30.4)$ & $14(28.0)$ & & 0.07 & \\
\hline & Mean (SD) & Mean (SD) & Mean (SD) & $U / \chi^{2}$ & Post hoc analysis \\
\hline Age & $38.8(11.5)$ & $42.6(13.8)$ & $38.2(12.0)$ & 2.8 & \\
\hline Education (years) & $14.2(1.7)$ & $14.3(2.5)$ & |4.2(।.8) & 0.8 & \\
\hline Duration of illness (years) & $7.8(5.8)$ & $9.6(8.9)$ & & $\mathrm{I}, 097.0$ & \\
\hline HDRS & $10.9(1.7)$ & $10.2(1.7)$ & $0.7(\mathrm{I} .0)$ & $111.7 * *$ & $W D=W o D>H C s$ \\
\hline BDI & $36.6(5.6)$ & $20.5(5.3)$ & $5.1(4.4)$ & $130.8 * *$ & $\mathrm{WD}>\mathrm{WoD}>\mathrm{HCs}$ \\
\hline BHS & $14.0(3.4)$ & $9.8(4.5)$ & $5.2(2.0)$ & $77.2^{* *}$ & $\mathrm{WD}>\mathrm{WoD}>\mathrm{HCs}$ \\
\hline
\end{tabular}

Notes: $* p<0.05 ; * * p<0.01$.

Abbreviations: BDI, Beck Depression Inventory; BHS, Beck Hopelessness Scale; HC, healthy control; HDRS, Hamilton Depression Rating Scale; MDD, major depressive disorder; WD, with a rating discrepancy; WoD, without a rating discrepancy. 


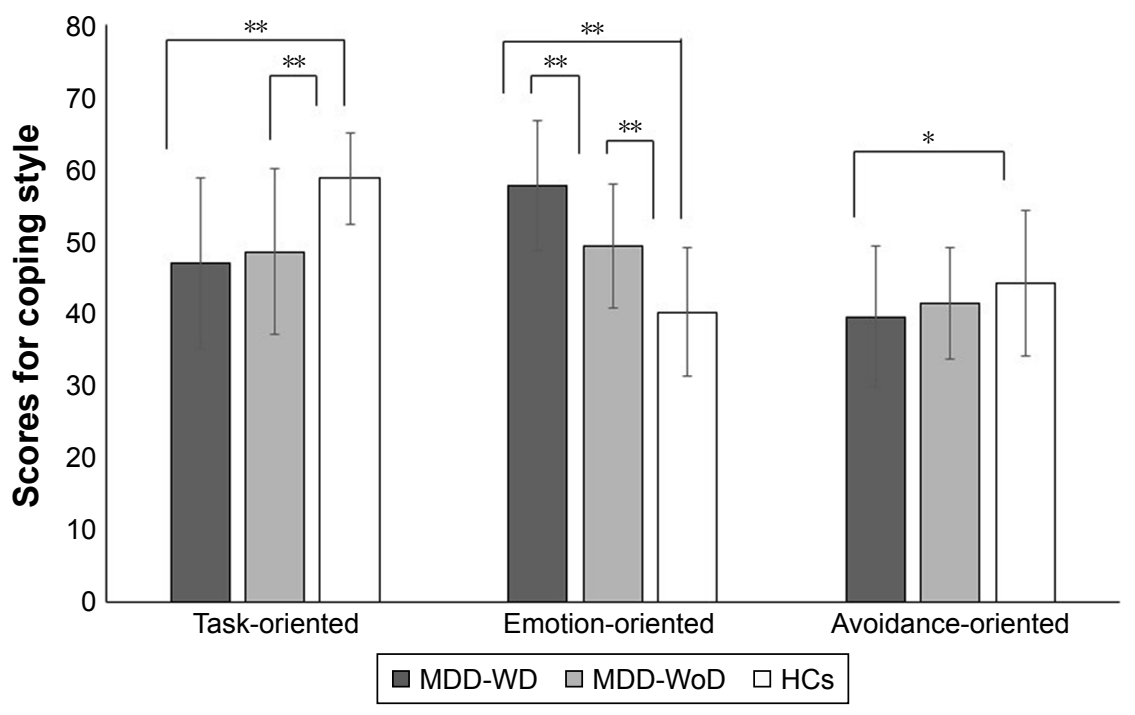

Figure I Results of the post hoc tests comparing the scores for the three coping styles between the three groups.

Note: $* P<0.05, * * p<0.01$.

Abbreviations: HC, healthy control; MDD, major depressive disorder; WD, with a rating discrepancy; WoD, without a rating discrepancy.

difference was observed between the two MDD patient groups $(P=0.44)$. The MDD-WD group also had significantly lower scores for avoidance-oriented coping than those of the HC group $(P=0.010)$. Interestingly, the two MDD patient groups demonstrated elevated scores for emotion-oriented coping compared with the $\mathrm{HC}$ group (both $P<0.001$ ), with the MDD-WD group demonstrating higher scores than those of the MDD-WoD group $(P<0.001)$.

\section{Predictor for rating discrepancy in patients with MDD}

Stepwise logistic regression analysis indicated that hopelessness, emotion-oriented coping, and a history of suicide attempts increased the likelihood of a discrepancy between self- and observer-rated depression severities (Table 2). In this analysis, hopelessness, emotion-oriented coping, and a history of suicide attempts had odds ratios of 1.31 (95\% CI 1.14-1.50), 1.11 (95\% CI 1.04-1.18), and 4.53 (95\% CI 1.15-17.9), respectively.

\section{Discussion}

This study investigated the association between coping styles and discrepancies in self- and observer-rated depression

Table 2 Prediction model for discrepancies between self- and observer-rated depression severities in patients with MDDa

\begin{tabular}{llll}
\hline Factor & $\boldsymbol{P}$-value & Odds ratio & $\mathbf{9 5 \%} \mathbf{C l}$ \\
\hline Hopelessness & $<0.00 \mathrm{I}$ & $\mathrm{I} .3 \mathrm{I}$ & $\mathrm{I} .14-\mathrm{I} .50$ \\
Emotion-oriented coping & $0.00 \mathrm{I}$ & $\mathrm{I} . \mathrm{II}$ & $\mathrm{I} .04-\mathrm{I} .18$ \\
History of suicide attempts & $0.03 \mathrm{I}$ & 4.53 & $\mathrm{I} .15-17.9$ \\
\hline
\end{tabular}

Note: aAnalyzed by stepwise logistic regression.

Abbreviation: MDD, major depressive disorder. severities in patients with MDD and how these coping styles might differ from those of HCs. Compared with HCs and patients in the MDD-WoD group, those in the MDD-WD group most frequently relied on emotion-oriented coping. Emotion-oriented coping emphasizes self-oriented reactions for coping with stress. These reactions involve emotional responses, where individuals blame themselves for being too emotional and ultimately become angry or tensed, as well as ruminative responses, where individuals obsessively brood over potential consequences. In addition, those with MDD-WD had significantly higher hopelessness scores than those in the MDD-WoD and control groups. Hopelessness is conceptualized as a set of cognitive schemas oriented toward negative views and expectations about the future. ${ }^{28}$ Previous studies have suggested that patients with MDD-WD exhibit greater anxiety ${ }^{30}$ and experience more suicide attempts, guilt, pessimism, and self-deprecation. ${ }^{31}$ Our results suggested that frequent use of emotion-oriented coping indicates a highly developed self-awareness of depression symptoms and could specify a unique psychopathological feature in a subset of patients with MDD.

It has been reported that cognitive dysfunction has a severe impact on a patient's ability to cope with the demands of daily living. ${ }^{32}$ In addition, persistent cognitive dysfunction in patients with MDD decreases their coping capacity. ${ }^{23}$ Consequently, depressed patients are inflexible in their use of coping styles. ${ }^{33}$ Previous studies have also reported that patients with MDD experience broad impairment in multiple aspects of executive functioning (eg, cognitive flexibility, behavioral inhibition, planning, and decision-making), ${ }^{34}$ 
resulting in rating discrepancy. ${ }^{35,36}$ Psychotherapies, such as cognitive behavioral therapy, problem-solving treatment, or interpersonal psychotherapy, are useful for increasing the usage of task-oriented coping and reducing depressive symptoms in undergraduates..$^{37,38}$ Our results also confirmed that patients with MDD rarely use task-oriented coping and emphasized the frequent use of emotion-oriented coping, at least in patients with a rating discrepancy. Indeed, both the MDD patient groups in this study used task-oriented coping less than that by the control group. This result is consistent with previous studies suggesting that task-oriented coping could be considered as an adaptive strategy in manageable situations. ${ }^{9-13}$ Overall, this finding highlights coping styles as potential targets for psychological intervention.

Patients with MDD-WD also relied less on avoidanceoriented coping compared with HCs, possibly because they had a strong self-awareness of their depression symptoms and no longer relied on avoiding the stressful situation. Previous investigations have reported that avoidance-oriented coping is related to extraversion, which is closely related to seeking social support. ${ }^{22,39}$ It is therefore possible that patients with MDD-WD are less motivated to rely on social support.

There were several methodological limitations to this study. First, we measured depression severity using BDI and HDRS for self- and observer-rated depression severities, respectively. These two scales measure alternative concepts of depression and possess several differences, thereby restricting our assessment of the real discrepancy between self- and observer appraisals. However, measuring various types of depressive symptoms may have helped to attain a more dynamic approach capable of evaluating associations among coping styles, hopelessness, and rating discrepancies. Second, because this study was cross-sectional, we could not determine the causal association between the severity of depressive symptoms and coping styles. Third, we excluded patients with MDD having alcohol/substance abuse or addiction. However, there is always a question of accuracy in patient's history when a condition may embarrass or otherwise compromise that patient, or when a patient is not aware of the condition. Because previous studies suggest that coping styles can be affected by confounders such as addiction or alcohol/substance abuse, our results may be limited in generalizability ${ }^{17,40}$ Finally, coping styles were measured using self-reports of the participants and their responses to specific social stressors. The association between subjective and objective interpretations of coping mechanisms and the respective association with depression warrants further study. Thus, future studies that involve a larger sample size are needed to verify our findings.

\section{Conclusion}

We found that frequent use of emotion-oriented coping was associated with discrepancies between self- and observerrated depression severities in individuals with MDD. Because a rating discrepancy in patients with MDD can be associated with serious outcomes such as increased tendency to attempt suicide, developing an effective coping strategy could be an effective psychotherapeutic intervention for limiting recurrent suicidal behavior. ${ }^{41}$ Planning to reduce the use of emotion-oriented coping mechanisms is one such example, which may reduce the feeling of hopelessness and risk of suicidal behavior in patients with MDD.

\section{Acknowledgments}

This study was partly supported by Grants-in-Aid for Scientific Research from the Japan Society for the Promotion of Science (No 25461792, No 22530776, and No 16K10229). We would like to thank Enago (https://www.enago.jp) for English language editing.

\section{Author contributions}

ET and NT designed the study, collected the data, undertook the statistical analyses, and wrote the manuscript. ET, NT, and WM collected the data. HO critically revised the text for important intellectual content. OS is the head of the laboratory and designed the study, wrote the protocols, and was involved in working on all drafts of the manuscript. All authors contributed toward data analysis, drafting and revising the paper and agree to be accountable for all aspects of the work.

\section{Disclosure}

The authors report no conflicts of interest in this work.

\section{References}

1. Rane LJ, Fekadu A, Wooderson S, Poon L, Markopoulou K, Cleare AJ Discrepancy between subjective and objective severity in treatmentresistant depression: prediction of treatment outcome. J Psychiatr Res. 2010;44(15):1082-1087.

2. Dunlop BW, Li T, Kornstein SG, et al. Concordance between clinician and patient ratings as predictors of response, remission, and recurrence in major depressive disorder. J Psychiatr Res. 2011;45(1):96-103.

3. Tsujii N, Akashi H, Mikawa W, et al. Discrepancy between self- and observer-rated depression severities as a predictor of vulnerability to suicide in patients with mild depression. J Affect Disord. 2014;161: 144-149.

4. Duberstein PR, Heisel MJ. Personality traits and the reporting of affective disorder symptoms in depressed patients. J Affect Disord. 2007;103(1-3): $165-171$.

5. Enns MW, Larsen DK, Cox BJ. Discrepancies between self and observer ratings of depression. The relationship to demographic, clinical and personality variables. J Affect Disord. 2000;60(1):33-41.

6. Schneibel R, Brakemeier EL, Wilbertz G, Dykierek P, Zobel I, Schramm E. Sensitivity to detect change and the correlation of clinical factors with the Hamilton Depression Rating Scale and the Beck Depression Inventory in depressed inpatients. Psychiatry Res. 2012;198(1):62-67. 
7. Kim EY, Hwang SS, Lee NY, et al. Intelligence, temperament, and personality are related to over- or under-reporting of affective symptoms by patients with euthymic mood disorder. J Affect Disord. 2013;148 $(2-3): 235-242$.

8. Endler NS, Parker JDA. Assessment of multidimensional coping: Task, emotion, and avoidance strategies. Psychol Assess. 1994;6(1):50-60.

9. Parker JDA, Endler NS. Coping with coping assessment: A critical review. Eur J Pers. 1992;6(5):321-344.

10. Johnsen BH, Laberg JC, Eid J. Coping strategies and mental health problems in a military unit. Mil Med. 1998;163(9):599-602.

11. Kendler KS, Kessler RC, Heath AC, Neale MC, Eaves LJ. Coping: a genetic epidemiological investigation. Psychol Med. 1991;21(2):337-346.

12. Rafnsson FD, Jonsson FH, Windle M. Coping strategies, stressful life events, problem behaviors, and depressed affect. Anxiety, Stress \& Coping. 2006;19(3):241-257.

13. Windle M, Windle RC, Strategies C. Coping strategies, drinking motives, and stressful life events among middle adolescents: associations with emotional and behavioral problems and with academic functioning. J Abnorm Psychol. 1996;105(4):551-560.

14. Alim TN, Feder A, Graves RE, et al. Trauma, resilience, and recovery in a high-risk African-American population. Am J Psychiatry. 2008;165(12): 1566-1575.

15. Liber JM, Faber AW, Treffers PD, van Loey NE, Style C. Coping style, personality and adolescent adjustment 10 years post-burn. Burns. 2008;34(6):775-782.

16. Rosenberg SJ, Peterson RA, Hayes JR. Coping behaviors among depressed and nondepressed medical inpatients. J Psychosom Res. 1987;31(5):653-658.

17. Wingo AP, Baldessarini RJ, Windle M. Coping styles: longitudinal development from ages 17 to 33 and associations with psychiatric disorders. Psychiatry Res. 2015;225(3):299-304.

18. Edwards MJ, Holden RR. Coping, meaning in life, and suicidal manifestations: examining gender differences. J Clin Psychol. 2001;57(12): 1517-1534.

19. Horwitz AG, Hill RM, King CA. Specific coping behaviors in relation to adolescent depression and suicidal ideation. J Adolesc. 2011;34(5):1077-1085.

20. Sugawara N, Yasui-Furukori N, Sasaki G, et al. Coping behaviors in relation to depressive symptoms and suicidal ideation among middleaged workers in Japan. J Affect Disord. 2012;142(1-3):264-268.

21. Svensson T, Inoue M, Charvat H, et al. Coping behaviors and suicide in the middle-aged and older Japanese general population: the Japan Public Health Center-based Prospective Study. Ann Epidemiol. 2014;24(3):199-205.

22. Uehara T, Sakado K, Sakado M, Sato T, Someya T. Relationship between stress coping and personality in patients with major depressive disorder. Psychother Psychosom. 1999;68(1):26-30.

23. Gonda X, Pompili M, Serafini G, Carvalho AF, Rihmer Z, Dome P. The role of cognitive dysfunction in the symptoms and remission from depression. Ann Gen Psychiatry. 2015;14:27.

24. Otsubo T, Tanaka K, Koda R, et al. Reliability and validity of Japanese version of the Mini-International Neuropsychiatric Interview. Psychiatry Clin Neurosci. 2005;59(5):517-526.
25. Rush AJ, First MB, Blacker D; American Psychiatric Association. Task Force for the Handbook of Psychiatric Measures. Washington, DC: American Psychiatric Association; 2008.

26. Williams JB. A structured interview guide for the Hamilton Depression Rating Scale. Arch Gen Psychiatry. 1988;45(8):742-747.

27. Beck AT, Steer RA, Ball R, Ranieri W. Comparison of Beck Depression Inventories-IA and -II in psychiatric outpatients. J Pers Assess. 1996;67(3):588-597.

28. Beck AT, Weissman A, Lester D, Trexler L. The measurement of pessimism: the hopelessness scale. J Consult Clin Psychol. 1974;42(6): 861-865.

29. Fukunishi I, Nakagawa T, Nakagawa H, et al. Validity and reliability of the Japanese version of the Stress and Coping Inventory. Psychiatry Clin Neurosci. 1995;49(4):195-199.

30. Corruble E, Legrand JM, Zvenigorowski H, Duret C, Guelfi JD. Concordance between self-report and clinician's assessment of depression. J Psychiatr Res. 1999;33(5):457-465.

31. Uher R, Farmer A, Maier W, et al. Measuring depression: comparison and integration of three scales in the GENDEP study. Psychol Med. 2008;38(2):289-300.

32. Hammar A, Ardal G. Cognitive functioning in major depression a summary. Front Hum Neurosci. 2009;3:26.

33. Gan Y, Zhang Y, Wang X, Wang S, Shen X. The coping flexibility of neurasthenia and depressive patients. Pers Individ Dif. 2006;40(5): 859-871.

34. Snyder HR. Major depressive disorder is associated with broad impairments on neuropsychological measures of executive function: a metaanalysis and review. Psychol Bull. 2013;139(1):81-132.

35. Rush AJ, Carmody TJ, Ibrahim HM, et al. Comparison of self-report and clinician ratings on two inventories of depressive symptomatology. Psychiatr Serv. 2006;57(6):829-837.

36. Akashi H, Tsujii N, Mikawa W, Adachi T, Kirime E, Shirakawa O. Prefrontal cortex activation is associated with a discrepancy between self- and observer-rated depression severities of major depressive disorder: a multichannel near-infrared spectroscopy study. J Affect Disord. 2015;174:165-172.

37. Kolovos S, Kleiboer A, Cuijpers P. Effect of psychotherapy for depression on quality of life: meta-analysis. Br J Psychiatry. 2016;209(6): 460-468.

38. Yamamoto A, Tsujimoto E, Taketani R, Tsujii N, Shirakawa O, Ono H. The Effect of Interpersonal Counseling for Subthreshold Depression in Undergraduates: An Exploratory Randomized Controlled Trial. Depress Res Treat. 2018;2018:4201897-6.

39. Amirkhan JH, Risinger RT, Swickert RJ. Extraversion: a "hidden" personality factor in coping? J Pers. 1995;63(2):189-212.

40. Corbin WR, Farmer NM, Nolen-Hoekesma S. Relations among stress, coping strategies, coping motives, alcohol consumption and related problems: a mediated moderation model. Addict Behav. 2013;38(4):1912-1919.

41. Turecki G, Brent DA. Suicide and suicidal behaviour. The Lancet. 2016;387(10024):1227-1239.
Neuropsychiatric Disease and Treatment

\section{Publish your work in this journal}

Neuropsychiatric Disease and Treatment is an international, peerreviewed journal of clinical therapeutics and pharmacology focusing on concise rapid reporting of clinical or pre-clinical studies on a range of neuropsychiatric and neurological disorders. This journal is indexed on PubMed Central, the 'PsycINFO' database and CAS,

\section{Dovepress}

and is the official journal of The International Neuropsychiatric Association (INA). The manuscript management system is completely online and includes a very quick and fair peer-review system, which is all easy to use. Visit http://www.dovepress.com/testimonials.php to read real quotes from published authors. 